\title{
Use of a 3D Model for Automatic Generation of Template Matching Algorithms
}

\author{
Marek Kočiško, Martin Pollák, Juraj Kundrík \\ Technical University of Košice, Faculty of Manufacturing Technologies with a seat in Prešov, \\ Bayerova 1, 08001 Prešov, Slovak Republic
}

\begin{abstract}
This paper deals with the current topic of computer vision which is used in quality control, accurate measurement or robot guidance. Templates are a key component of template matching algorithms. Creating them is a complex and demanding process. The authors of the paper try to describe the possibilities of automated template generation from information that is already available about the product. A digital version in the CAD system exists of virtually every product nowadays. An experiment has shown that with the use of this information, it is possible to create a template that will replace templates created through standard procedures.
\end{abstract}

Keywords - computer vision, template matching, digital twin, quality control, artificial intelligence.

\section{Introduction}

Computer vision is an essential part of an automated manufacturing process. It is used for robot guidance, quality control and production line management. One of the oldest and most frequently used technologies is template matching. It is used to detect the presence of an object or the location of an object in the examined space.

DOI: 10.18421/TEM103-45

https://doi.org/10.18421/TEM103-45

Corresponding author: Marek Kočiško,

Technical university of Košice, Faculty of Manufacturing Technologies with a seat in Prešov, Slovak Republic.

Email: marek.kocisko@tuke.sk

Received: 15 July 2021.

Revised: 10 August 2021.

Accepted: 16 August 2021.

Published: 27 August 2021.

(cc) BY-NC-ND]C 2021 Marek Kočiško, Martin Pollák \& Juraj Kundrik; published by UIKTEN. This work is licensed under the Creative Commons Attribution-NonCommercialNoDerivs 4.0 License.

The article is published with Open Access at www.temjournal.com
It is about matching the template to a part of the scene. A template is an image of the sought product, or a part of it.

A scene is an image of the environment in which the presence and location of a template is searched for using a suitable algorithm. The place with the greatest match between the template and the scene is the matched region. [1]

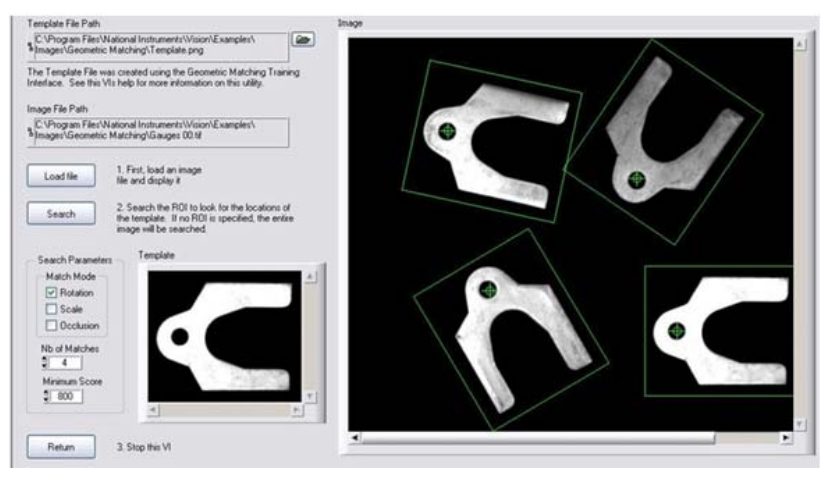

Figure 1. Template matching application for industry [2]

Figure 1. is an example of a template matching application in mechanical engineering production. The small image on the left is the product template and on the right is the image of the scene with the product positions that have been identified with the use of the template.

To detect an object in template matching, it is important to create a pattern of the "ideal product", i.e., a template that contains an image of the sought object. To create it, a photograph of the examined space, called a scene, is usually used. The template is thus generated by cropping that part of the scene that contains the area of interest. By comparing the template and the newly created scene, it is possible to verify the existence and position of the sought object. This procedure requires that the recognized object exists, is placed in the scene and the photograph of the scene is subsequently processed.

By creating a template without the existence of the sought product and without it being placed in the scene, it is possible to simplify and speed up template drafting. The paper deals with a template-generating algorithm from a 3D model in the CAD system. With this procedure, it is not necessary to place the product 
in the scene, which simplifies and speeds up the process of deploying template matching in production. Without creating a template from a scene photograph, it is possible to automate the process of template generation, which would allow this procedure to be deployed in an automated production process.

In addition to designing the procedure for template generation, it is necessary to verify the possibilities of the template generated in this way when recognizing the scene in the real environment in simulating problems solved with the use of template matching. In the conducted experiments, the properties of the generated templates were verified using the functions of the openCV library.

\section{State of Art}

Template matching is one of the oldest technologies in the field of computer vision. However, research in this area is still ongoing. The original algorithm, called "Sum of Absolute Differences" [3], is based on comparing the sought image with the view, the scene, pixel by pixel.

The difference function that compares the individual pixels is described in formula (1).

$$
\text { dif } f\left(x_{t}, y_{t}, x_{s}, y_{s}\right)=\left|S\left(x_{s}, y_{x}\right)-T\left(x_{t}, y_{t}\right)\right|
$$

where:

$\mathrm{T}\left(\mathrm{x}_{\mathrm{t}}, \mathrm{y}_{\mathrm{t}}\right)=$ light intensity in the template, at the point determined by the $\mathrm{x}_{\mathrm{t}}, \mathrm{y}_{\mathrm{t}}$ coordinates,

$\mathrm{S}\left(\mathrm{x}_{\mathrm{s}}, \mathrm{y}_{\mathrm{s}}\right)=$ light intensity in the scene, determined by the $\mathrm{x}_{\mathrm{s}}, \mathrm{y}_{\mathrm{s}}$ coordinates.

The total correlation $\mathrm{K}$ between the template and the part of the scene determined by points $\mathrm{x}$ and $\mathrm{y}$ is then calculated using formula (2):

$$
K(x, y)=\sum_{i=0}^{T r o w s} \sum_{j=0}^{T c o l s} \operatorname{dif} f(x+j, y+i, j, i)
$$

where:

Trows $=$ number of template rows,

Tcols $=$ number of template columns .

$\mathrm{x}, \mathrm{y}=$ coordinates of the point for which the correlation is calculated.

Evaluation of $\mathrm{K}$ delimits a part of the scene that is most similar to the template. The $\mathrm{x}, \mathrm{y}$ points determine the location of the sought template in the scene. [1]

The input for this algorithm is two images. The larger one captures a scene that may be, for example, a view of a product. The second image, the template, contains only the object the occurrence of which needs to be found in the scene. The $\mathrm{K}$ value determines the correlation between the template and the individual scene parts. By interpreting the $\mathrm{K}$ value and the coordinates that belong to it, it is possible to determine the position of the sought object in the scene.
This algorithm is slow and not very robust. To work properly, the template must be in the same scale and rotated in the same direction as the object in the scene. At the same time, similar lighting conditions must be observed during implementation as in template generation.

Therefore, most research has focused on finding faster and more robust algorithms. With powerful computers increasingly more available, the problem is not computing power, but rather low algorithm robustness. Research in the field of computer vision has led to two basic directions of addressing the problems of template matching algorithm robustness.

Feature based algorithms look for similarity between a template and a scene based on the properties of the template, such as colour, shape, or texture. These algorithms select and compare the characteristics of the template and the scene, the examples are Object recognition from local scaleinvariant features [4] and SURF: Speeded Up Robust Features [5]. They use one template for recognition and can also address its rotation or resizing. They work properly for tasks with a lot of detail, so they are not suitable for searching for simple shapes such as holes or screws.

The algorithm described in Rapid Object Detection using a Boosted Cascade of Simple Features [6] is based on the above-mentioned algorithms. When multiple templates are used, statistical methods are deployed to create a "universal recognition template". A similar procedure is used in the paper titled Multicolumn Deep Neural Networks for Image Classification [7], where neural network technology is used instead of statistical methods. To function correctly, this procedure also requires several tens or hundreds of "templates", i.e., images of the object sought. Images are used to create algorithm implementations. The advantage of these procedures is a robust system, the disadvantage is a timeconsuming implementation period. In production, these algorithms are used for complex problems such as fault detection, bin picking problems and the like.

Template-based algorithms look for a correlation between the template as a whole. By comparing the template and the part of the scene, the correlation between the template and the scene is established. There are several ways to compare the template and the scene. Currently, research on these algorithms is focused on improving the algorithm robustness. In the work titled Fast and robust template matching with majority neighbour similarity and annulus projection transformation [8], which, in addition to the correlation between the points of the template and the scene, also evaluates the correlation of the surrounding points. It then determines the position of the template in the scene based on the highest match. The result is a highly robust system that also recognizes similar and rotated template placements. 


\section{Creation of the Template for the Template Matching}

\subsection{Washers}

For the template matching to work properly, it is necessary that the template has the same shape, size and direction of rotation as its copy in the scene. The problem of differing direction of the template position and that of the image in the scene is usually resolved by rotating the template during the correlation evaluation. The use of centrally symmetrical washers eliminates the need to rotate the template. The washers simplify the creation of a program that will be used to verify the quality and usability of the template generated. [9]

Minimal differences between the individual washer types allow for better testing of the resolution accuracy of each template matching method. Four types of washers were used in the experiment, for which a template was generated. Figure 2. is a section of the washer drawing. The drawing was created according to dimensions of real washers directly in the CAD system. The fifth type was used as a reference sample to test the recognition correctness. No model has been created for this type. In addition to washers made of galvanized sheet steel, washers made of brass and stainless steel were also used in the experiment.

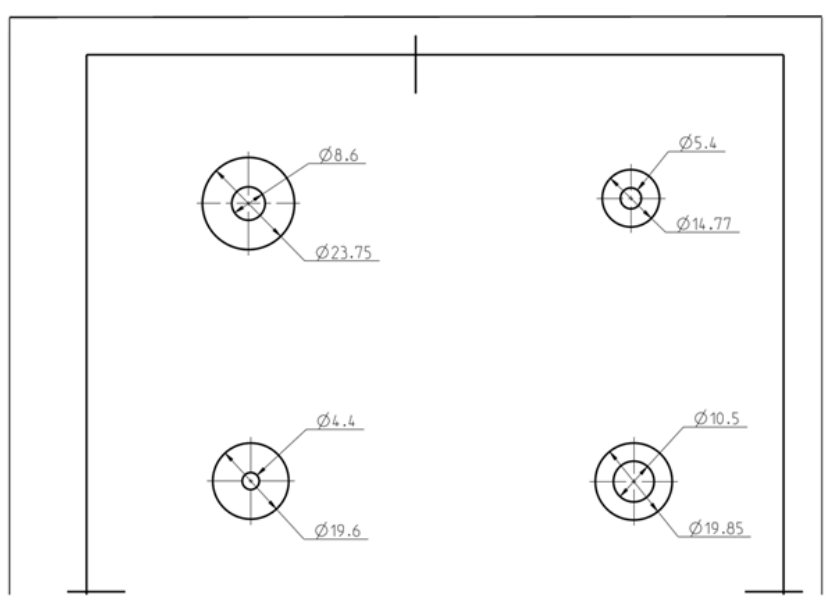

Figure 2. Technical drawing of used washers

\subsection{Camera}

Industrial cameras with interchangeable lenses are used in practical computer vision applications. When designing an industrial application, lens with the parameters such as aperture or depth of field, matching the specifications, is selected. Appropriate lens parameters reduce errors when converting an analogue image to a digital log. This distortion affects the accuracy and robustness of the application. The choice of camera is, therefore, key to the application's proper functioning. [10]
The size of the template must match the size of the object sought in the scene. Therefore, the accuracy of converting the camera image to digital $\log$ is important when generating a template and then recognizing it in the scene.

The selected SVGA resolution ( $800 \times 600$ pixels) is smaller than industry standards. This increased error caused by the inaccuracy of conversion of the washer dimensions to pixels in the digital $\log$, and thus it was possible to verify the robustness of the generated template.

\subsection{CAD System PTC CREO}

The family of Creo programs, is one of the most used and most comprehensive sets of CAx programs. It contains classic tools for 2D and 3D modelling, parametric modelling, programs for $2 \mathrm{D}$ and $3 \mathrm{D}$ visualizations.

The Material Appearance subsystem is used to display the model, which enables setting material properties such as colour, brightness, texture, etc. When creating the model, it is possible to assign material to it and get a more realistic view of the model.

Creo does not have a direct program for generating a template suitable for computer vision, it was necessary to create such program. The program used functions from the API interface and the Creoson library. All programs were created using the Python programming language. This enables an automatic template generation process.

\subsection{OpenCV Library}

OpenCV is a freely available library of functions focused on real-time computer vision. It is the most widely used, freely distributable library for this type of task. Versions for C ++, Java, Python, Matlab and Javascript are available. A Python implementation was used in the experiment.

The openCV library has three methods applied to template matching to determine the correlation between a scene and a template:

- square difference,

- cross correlation,

- correlation coefficient.

To better validate the template, all three implementations of the search algorithm were used in the testing.

Most research in the field of template matching takes place in the field of computer science and therefore focuses mainly on improving the search algorithms. Ordinary photographs are used to test algorithms during research. A template is usually created from a section of a part of the scene. 
In the paper titled Multi-template matching algorithm for cucumber recognition in natural environment [11], the process of creating a template in agricultural production is designed and tested in order to create a robust system for recognizing natural shapes. The process involves generating templates derived from the base template by rotating or shrinking. The implementation algorithm then sequentially applies each template to the scene under examination. Such an increase in robustness results in a deceleration, which is not an obstacle in this application of the cucumber recognition algorithm.

In the Cross-correlation Template Matching for Liver Localization in Computed Tomography [12], the authors also deal with the creation of a template for liver recognition in $\mathrm{CT}$ images. To solve this problem, it was necessary to create two templates that displayed the liver from the top and the side view.

\subsection{Experimental Algorithm}

The purpose of the experiment was to verify the possibilities of creating a template from a CAD model. Design and create a program that generates a template from a CAD system model. Then test this template on the scene analysis. Washers were placed in the scene, the CAD models of which were used to generate the template and were being recognized by the generated template.

The experiment itself consisted of three steps:

a) scale calculation (i.e., how many pixels in the image correspond to one millimetre)

b) template generation from a CAD system

c) recognition testing using template matching

\subsection{Calculation of the Scale}

For the template matching algorithm to work properly, it is necessary to prepare a template in the same scale as in the scene. When generating a template directly from a scene image using a cutout, the template is automatically generated in the correct scale. When generating a template from a CAD program, it is necessary to scale the template. In order to automate the whole process, a program was created that established a scale using an object of a known size. The program is thus used to automatically calibrate the process of template generation.

When calibrating the scene, a calibre of known size was the only object placed in the scene. The image of the scene was processed using computer vision procedures and the size of the calibration object was established in pixels. The scale itself was calculated using formula (3).

$$
\text { scale }=\frac{s_{p}}{s_{m}}
$$

where:

$\mathrm{s}_{\mathrm{p}}=$ number of pixels,

$\mathrm{s}_{\mathrm{m}}=$ size of the object in $\mathrm{mm}$.

Figure 3. shows the scene to be calibrated and the manner of establishing the size of the calibration object. A 2-euro coin was used in the experiment.

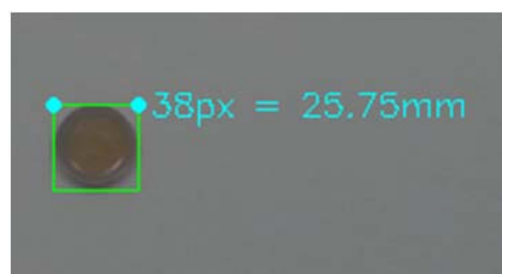

Figure 3. Calibration picture

\subsection{Template Generation}

The algorithm of the program for generating the template is shown in Figure 4. Using the functions of the creopyson library and the Creo API library, the program uploads the file and sets the view of the component. It then exports the view of the 3D model image Error: Reference source not found as a PNG graphic file. Using the functions of the API interface, Creo identifies the described rectangle, which does not contain anything but the washer. This rectangle is used to crop a template from the exported image. The size of the cropped rectangle corresponds to the size of the product in millimetres. Using the scale from the previous step, it is then possible to transform the template to the dimension corresponding to the scene.

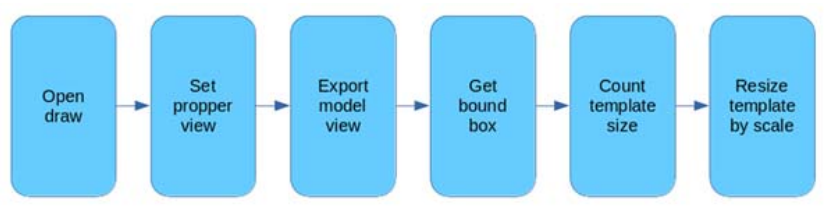

Figure 4. Template algorithm

CAD systems also have modules for photorealistic display of the product rendering modules. The Appearance module was used to visualize the washers when creating a product model in the experiment. Figure 5. shows front view of washer model. This module simulates the appearance of the impression of the model depending on the model material. In order to achieve the greatest possible similarity between the model and the product, CAD systems contain definitions of materials used in production. In the experiment were used the definitions of materials contained in the Creo system and corresponding to the washers. For washers 1 to 4 , it was galvanized steel, for washer 5 , it was brass, and for washer 6 stainless steel. 


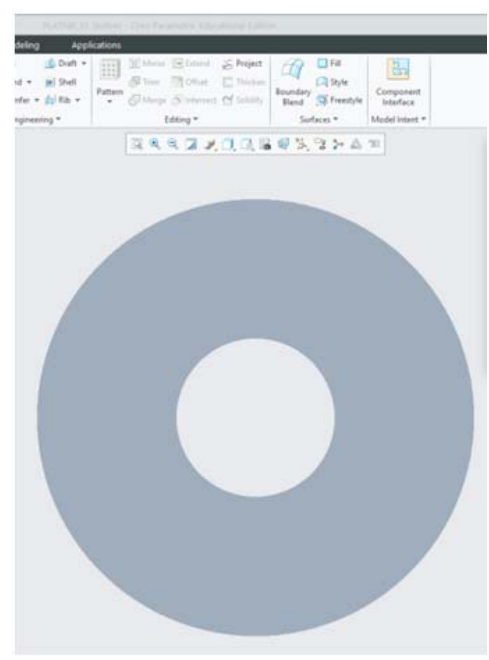

Figure 5. Washer model front view shown by Appearance module

The mathematical principles of template matching algorithms show that better results will be achieved with greater contrast between the model and the background. Two templates were created for each washer type differing in background colour. Both the model material and the background can be set in Creo by a modeler, or they can be defined in the process of template generation using the API interface.

Images of templates created during the experiment are shown in Figure 6.

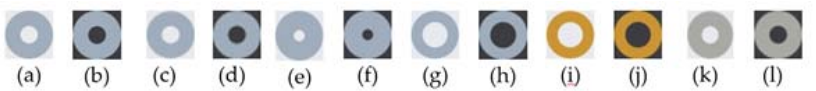

Figure 6. Templates created from CAD model. With normal and contrast background for all types of washers. Washer 1: (a), (b); Washer 2 (c), (d); Washer 3 (e), (f);

Washer 4 (g), (h); Washer 5 (i), (j); Washer 6 (k), (l)

In practice, templates created from a photograph cutout are used most often. Such templates were also used in the experiment for searching. Figure 7. shows the templates created as a cutout and used in the experiment to verify the correctness of the search algorithm applications.

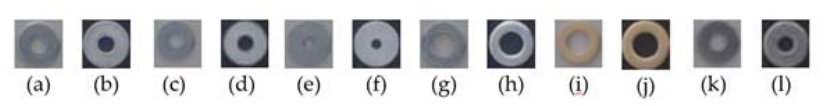

Figure 7. Templates cropped from the scene picture. With normal and contrast background for all types of washers.

Washer 1: (a), (b); Washer 2 (c), (d); Washer 3 (e), (f);

Washer 4 (g), (h); Washer 5 (i), (j); Washer $6(k)$, (l)

\section{Realization Experiments and Testing}

\subsection{Recognition Process}

Comparing the scene and the template creates a map of the comparison results. Its visualization is in Figure 8., the highest correlation corresponds to the brightest point. The highest correlation value is the recognized location of the template in the scene and is indicated by a red square in the scene. A successful recognition represented a designation that corresponded to the type of washer of the washer sought.
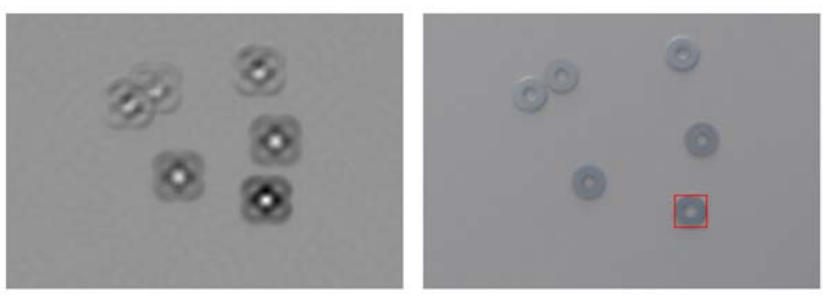

Figure 8. Example of map of comparison results (a), detected point in original scene (b). Cross-correlation algorithm and washer 2

The aim of the experiment was to compare the success of the 3D and the cropped template for the most commonly used template matching algorithms. For each washer type, templates were created in both ways and the recognition success was evaluated.

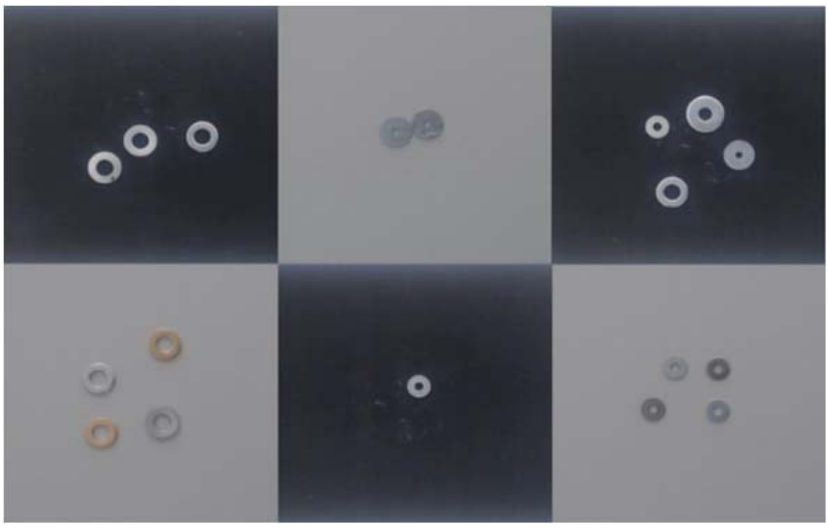

Figure 9. Examples of recognition scenes

The templates were tested on almost 200 photographs of the scenes the examples of which are shown in Figure 9.

\subsection{Experiment Results}

More than 500 searches were evaluated in the experiment, and the results are plotted in graphs according to the type of the algorithm used. The graphs in the image are in Figure 10. for the Correlation Coefficient algorithm, Figure 11. shows the success graphs for the Cross Correlation algorithm and Figure 12. shows the results for the Sum of Square Differences algorithm. In the picture on the left, the graph shows the results for a noncontrasting (light) background, on the right for a contrasting, black background.

The value at the top of the graph is the ratio of successfully recognized positions and all the scenes tested. The type of washer is marked w1 to w6. The values for the template generated from a section of the scene are marked in blue. Red value for templates is generated from a $3 \mathrm{D}$ model. 

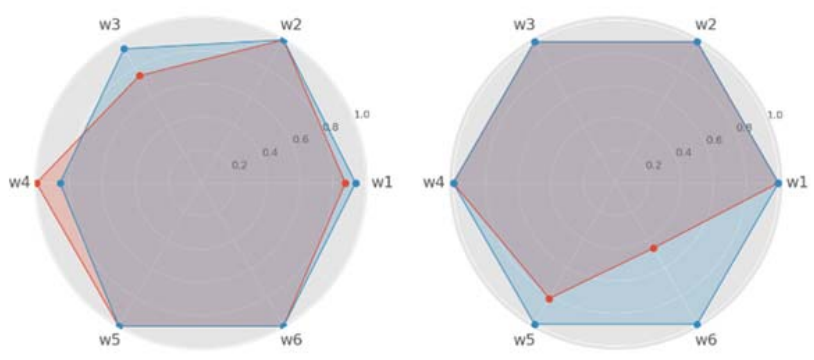

Figure 10. Ratio of recognized all position for every washer type for Correlation Coefficient algorithm with normal background (a) and contrast background (b). $3 D$ model template red, cropped template blue.
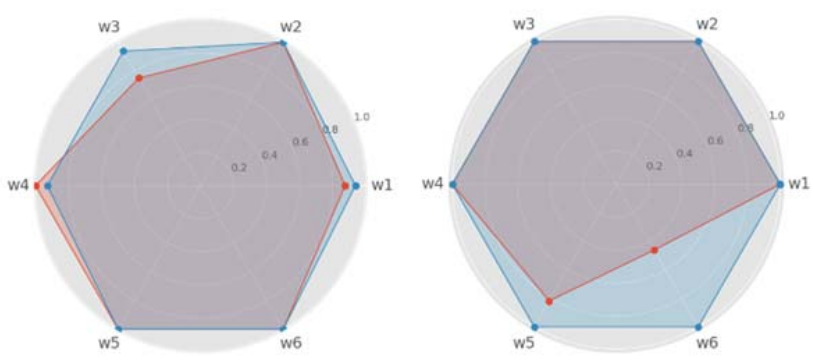

Figure 11. Ratio of recognized all position for every washer type for Cross Correlation algorithm with normal background (a) and contrast background (b). 3D model template red, cropped template blue.
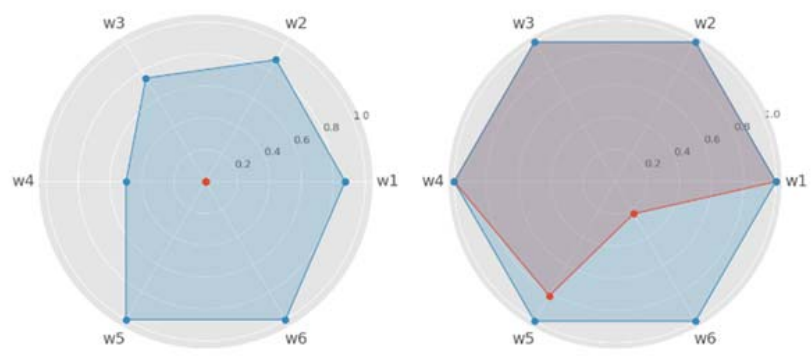

Figure 12. Ratio of recognized all position for every washer type for Sum of Square Differences algorithm with normal background (a) and contrast background (b). 3D model template red, cropped template blue.

Simply put, the larger the areas covered by the graphs, the more accurate the recognition for a given algorithm and template. If the red and blue areas overlap, the search result does not depend on the template type.

The Sums of Square Differences algorithm has the worst search results. The graphs in Figure 12. show a strong dependence on the contrasting background. This algorithm is used very rarely in special cases.

The Cross Correlation and Correlation Coefficient algorithms have virtually the same search results for both template generation methods. These algorithms are most commonly used in template matching implementations.

The experiment showed the expected positive effect of the contrast background. The contrasting background improves the recognition success in case of galvanized washers w1 - w4. For both template types. In case of the w5 brass washer, it slightly worsened the recognition of the template generated from the 3D model.

Ambiguous results for the stainless-steel washer w6 can be attributed to various causes. Inaccurately created glossy surface of the stainless-steel washer using the material definition in Creo, Inappropriate lighting of the scene, which creates reflections on the stainless-steel washer.

The experiment showed that it is possible to create templates for template matching also with the use of the CAD system functions to display the model. When implementing such procedure, it is most important to verify the behaviour of the material model defined in the CAD system in a real environment.

The results of the experiment show that it is possible to use a $3 \mathrm{D}$ product model and an API interface for automated template generation. This makes it possible to simplify the process of implementing template matching in production.

\section{Conclusion}

The paper is based on the digital twin principles. It deals with the use of information about the product, which originate during its design, and their use in the service activities of the production process. The automation of the production process is not possible without computer vision technologies, which enable the product handling and inspection [13], [14]. It explores other possibilities of using CAD systems, the special functions of 3D model generation.

Template matching is a computer vision technology widely used in production. The templates themselves are an important part of implementing this technology. Such application cannot work without a correct template [15]. Today, the outputs of 3D models from CAD software are so much similar to the digital image from a video camera. The paper describes the algorithm and principles of template generation from a 3D model in a CAD program. To generate a template, all that is needed is the simple use of the function for generating a realistic 3D model and convert it to a 2D image. The API interface of a common CAD system provides functions that enable the automation of template generation.

Part of the thesis are experiments that verify the possibilities of practical use of the resulting template. The experiment showed that a template created in a $3 \mathrm{D}$ model of a drawing can replace a template created from a photograph of a real product. In this way, the entire drafting and deployment of the template can be automated. This will make it possible to respond automatically to changes in the 
production process, which will increase the production process flexibility and efficiency. The paper contains a comprehensive guide to addressing problems in the implementation of automatically generated templates.

In addition to correlation, the template matching output corresponds to the position of the template in the scene, therefore, it is possible to use this output in product handling. Based on its location in the scene, it is possible to control the robot movement. The change in the product shape can thus be automatically transferred to the system of robot navigation [16], [17]. Another application is automated production inspection. For example, verifying the presence of holes in intended locations. The benefit of such procedure is an automatic response to changes in the location or shape of the inspected components.

Product changes in CAD system can now be transferred directly to production using a $\mathrm{CNC}$ program. The paper shows the possibilities of automating service activities that are part of the automated production process. Procedures derived from such process will also find use in the design of automated production cells. Such production cell would be able to automatically produce certain product types solely on the basis of information from the product technical documentation.

\section{Acknowledgements}

This paper has been elaborated in the framework of the project VEGA 1/0051/20 and project KEGA 038TUKE$4 / 2021$.

\section{References}

[1]. Sonka, M., Hlavac, V., \& Boyle, R. (2014). Image processing, analysis, and machine vision. Cengage Learning.

[2]. Bergamini, L., Sposato, M., Peruzzini, M., Vezzani, R., \& Pellicciari, M. (2018, July). Deep learningbased method for vision-guided robotic grasping of unknown objects. In Transdisciplinary Engineering Methods for Social Innovation of Industry 4.0: Proceedings of the 25th ISPE Inc. International Conference on Transdisciplinary Engineering (Vol. 7, p. 281).

[3]. Byne, J. H. M., \& Anderson, J. A. D. W. (1998). A CAD-based computer vision system. Image and Vision Computing, 16(8), 533-539.

[4]. Lowe, D. G. (1999, September). Object recognition from local scale-invariant features. In Proceedings of the seventh IEEE international conference on computer vision (Vol. 2, pp. 1150-1157). IEEE.
[5]. Bay, H., Tuytelaars, T., \& Van Gool, L. (2006, May). Surf: Speeded up robust features. In European conference on computer vision (pp. 404-417). Springer, Berlin, Heidelberg.

[6]. Viola, P., \& Jones, M. (2001, December). Rapid object detection using a boosted cascade of simple features. In Proceedings of the 2001 IEEE computer society conference on computer vision and pattern recognition. CVPR 2001 (Vol. 1, pp. I-I). Ieee.

[7]. Ciregan, D., Meier, U., \& Schmidhuber, J. (2012, June). Multi-column deep neural networks for image classification. In 2012 IEEE conference on computer vision and pattern recognition (pp. 3642-3649). IEEE.

[8]. Lai, J., Lei, L., Deng, K., Yan, R., Ruan, Y., \& Jinyun, Z. (2020). Fast and robust template matching with majority neighbour similarity and annulus projection transformation. Pattern recognition, 98, 107029 .

[9]. Goshtasby, A. (1985). Template matching in rotated images. IEEE Transactions on Pattern Analysis and Machine Intelligence, (3), 338-344.

[10]. De Villiers, J. P., Leuschner, F. W., \& Geldenhuys, R. (2008, November). Centi-pixel accurate real-time inverse distortion correction. In Optomechatronic Technologies 2008 (Vol. 7266, p. 726611). International Society for Optics and Photonics.

[11]. Bao, G., Cai, S., Qi, L., Xun, Y., Zhang, L., \& Yang, Q. (2016). Multi-template matching algorithm for cucumber recognition in natural environment. Computers and Electronics in Agriculture, 127, 754-762.

[12]. Leydon, P., O'Connell, M., Greene, D., \& Curran, K. M. (2019, July). Cross-Correlation Template Matching for Liver Localisation in Computed Tomography. In The 2019 Irish Machine Vision and Image Processing Conference (IMVIP 2019), Technological University Dublin, 28-30 August 2019.

[13]. DOBRANSKY, J., BOTKO, F., \& VOJNOVA, E. (2016). Monitoring of production quality for plastic component. measurement, 10, 30.

[14]. Panda, A., Nahornyi, V., Pandová, I., Harničárová, M., Kušnerová, M., Valíček, J., \& Kmec, J. (2019). Development of the method for predicting the resource of mechanical systems. The International Journal of Advanced Manufacturing Technology, 105(1), 1563-1571.

[15]. Stejskal, T., Dovica, M., Svetlik, J., \& Demec, P. (2019). Experimental assessment of the static stiffness of machine parts and structures by changing the magnitude of the hysteresis as a function of loading. Open Engineering, 9(1), 655-659.

[16]. Zaborowski, T., Panda, A., Androvic, L., \& Onofrejova, D. (2019). Robots and cobots, main differences. Studia i Materiaty, 39(6), 4-8.

[17]. Ondočko, Š., Svetlík, J., Šašala, M., Bobovský, Z., Stejskal, T., Dobránsky, J., ... \& Hrivniak, L. (2021). Inverse Kinematics Data Adaptation to Non-Standard Modular Robotic Arm Consisting of Unique Rotational Modules. Applied Sciences, 11(3), 1203. 